\title{
A FUNCTIONAL CENTRAL LIMIT THEOREM IN DIOPHANTINE APPROXIMATION
}

\author{
JORGE D. SAMUR
}

(Communicated by William D. Sudderth)

\begin{abstract}
A functional central limit theorem is proved for the number of solutions $(p, q)$ of the inequality $|q \omega-p|<f(q) q^{-1}, q \leq n$ (respectively $0<q \omega-p<f(q) q^{-1}, q \leq n$ ) for some functions $f$ having a positive limit.
\end{abstract}

\section{INTRODUCTION AND STATEMENT}

Let $\Omega$ be the set of irrational numbers in $(0,1)$ and let $\mathscr{B}$ be the class of its Borel subsets.

Given a function $f(q)$ of the positive integer $q$ with $0<f(q) \leq 1 / 2$, define

$$
\begin{aligned}
\Lambda_{n}(\omega)=\Lambda_{f, n}(\omega) & =\#\left\{(p, q) \in \mathbf{N} \times \mathbf{N}^{*}:|q \omega-p|<\frac{f(q)}{q}, 1 \leq q \leq n\right\} \\
& =\#\left\{q \in \mathbf{N}^{*}:\langle q \omega\rangle<\frac{f(q)}{q}, 1 \leq q \leq n\right\},
\end{aligned}
$$

where $\langle\alpha\rangle$ is the distance between $\alpha$ and the nearest integer, and

$$
\begin{aligned}
\Lambda_{n}^{+}(\omega)=\Lambda_{f, n}^{+}(\omega) & =\#\left\{(p, q) \in \mathbf{N} \times \mathbf{N}^{*}: 0<q \omega-p<\frac{f(q)}{q}, 1 \leq q \leq n\right\} \\
& =\#\left\{q \in \mathbf{N}^{*}: q \omega-[q \omega]<\frac{f(q)}{q}, 1 \leq q \leq n\right\}
\end{aligned}
$$

( $n \geq 1, \omega \in \Omega$; throughout the paper [ $\cdot]$ denotes the integer part of a real number).

Assume that $\sum f(q) q^{-1}$ diverges. For fairly general $f$, the theorems of LeVeque, Erdös, and Schmidt ([5], [3], [9], [4]) show that $\Lambda_{f, n} \sim 2 \sum_{q \leq n} f(q) q^{-1}$ $(=b(n)$, say) a.e., thus improving a classical theorem of Khintchine. Philipp $[6, \S 3]$ proved, in accordance with a conjecture of LeVeque [5b], the asymptotic normality of $\Lambda_{f, n}$ (explicitly, that $b(n)^{-1 / 2}\left(\Lambda_{f, n}-b(n)\right)$ tends to the standard

Received by the editors August 25, 1989.

1980 Mathematics Subject Classification (1985 Revision). Primary 11K60; Secondary 60F05, $60 \mathrm{~F} 17$.

Key words and phrases. Diophantine approximation, functional central limit theorem, invariance principle, continued fraction expansion, mixing random variables. 
normal law) and that of other related quantities, for $f$ satisfying, besides other conditions, $\lim _{q} f(q)=0$; also, [6] contains results of law of iterated logarithm type in this case (in addition, the theorems in [7] - see the introduction-would yield almost sure invariance principles).

Moreover, in [6, p. 43] it is asserted that, with appropriate norming constants, similar results would continue to hold for nonincreasing functions $f$ for which $f(q)=c+O\left((\log q)^{-1-\varepsilon}\right)$ for some constants $\varepsilon>0$ and $0<c<10^{-3}$.

In this paper, we prove a functional central limit theorem (invariance principle) for $\Lambda_{n}\left(\Lambda_{n}^{+}\right)$under a slightly weaker assumption on $f$.

We fix some notation. We will denote by $\lambda$ the Lebesgue measure on $(\Omega$, $\mathscr{B})$. If $\rho$ is any probability measure on this space we shall write $E_{\rho}$ for the corresponding expectation operator and $\mathscr{L}_{\rho}(\xi)$ for the law of a random element $\xi$ defined on $(\Omega, \mathscr{B}, \rho)$; moreover, if $\rho$ is absolutely continuous with respect to $\lambda$, we shall write $\rho \ll \lambda$. For the concepts of Skorohod space, Wiener measure, and weak convergence of probability measures, which shall be denoted by $\rightarrow_{w}$, we refer to [2].

The following result has a version in $C([0,1])$.

Theorem. Let $f(q)$ be a nonincreasing function of the positive integer $q$ such that $0<f(q) \leq 1 / 2$ and

$$
f(q)=c+o\left(\frac{\log (\log q)}{\log q}\right) \text { for some constant } 0<c \leq 1 / 2
$$

Consider the random functions

$$
\begin{aligned}
& \xi_{n}(t)=\left(\frac{\pi^{2} / 6}{2 c \log n \log (\log n)}\right)^{1 / 2}\left\{\Lambda_{f,\left[n^{t}\right]}-2 c \log \left[n^{t}\right]\right\}, \\
& \xi_{n}^{+}(t)=\left(\frac{\pi^{2} / 6}{c \log n \log (\log n)}\right)^{1 / 2}\left\{\Lambda_{f,\left[n^{t}\right]}^{+}-c \log \left[n^{t}\right]\right\},
\end{aligned}
$$

$t \in[0,1], n \geq 3$. Then for any $\rho \ll \lambda, \mathscr{L}_{\rho}\left(\xi_{n}\right) \rightarrow_{w} W$ and $\mathscr{L}_{\rho}\left(\xi_{n}^{+}\right) \rightarrow_{w} W$ in the Skorohod space $D([0,1]), W$ being standard Wiener measure.

The proof involves the continued fraction expansion $\left[0, a_{1}, a_{2}, \ldots\right]$ of a number in $\Omega$, and a limit theorem for mixing random variables with infinite variances. As is suggested by an estimation made in [4, p. 35], we compare $\Lambda_{n}$ with (random) partial sums of $\left\{a_{j}^{1 / 2}\right\}$ and we apply the functional limit theorem proved for these sums in [8, Example 2.14.1] (see 2.15 there for references). The comparison is carried out starting from relation (5) below (note that when $f \equiv$ $c$, we have $r_{j}=\left[\left(c u_{j}\right)^{1 / 2}\right]$ a.e.) and using Lemma 1 (to show that $\tilde{r}_{j} \approx\left(c a_{j}\right)^{1 / 2}$, where $\tilde{r}_{j}$ is a suitable approximation of $r_{j}$ ), which follows from arguments of Ibragimov and Billingsley. 


\section{PROOF}

Given $\omega \in \Omega$, let $\omega=\left[0, a_{1}(\omega), a_{2}(\omega), \ldots\right]$ be its simple continued fraction expansion. We will deal also with the following quantities:

$$
\begin{aligned}
& x_{n}(\omega)=\left[a_{n}(\omega), a_{n+1}(\omega), \ldots\right], \\
& p_{0}(\omega)=0, p_{1}(\omega)=1, p_{n}(\omega)=a_{n}(\omega) p_{n-1}(\omega)+p_{n-2}(\omega) \quad \text { if } n \geq 2, \\
& q_{0}(\omega)=1, q_{1}(\omega)=a_{1}(\omega), q_{n}(\omega)=a_{n}(\omega) q_{n-1}(\omega)+q_{n-2}(\omega) \quad \text { if } n \geq 2, \\
& y_{n}(\omega)=\frac{q_{n}(\omega)}{q_{n-1}(\omega)}=\left[a_{n}(\omega), a_{n-1}(\omega), \ldots, a_{1}(\omega)\right], \\
& \frac{1}{u_{n}(\omega)}=\left|\omega-\frac{p_{n-1}(\omega)}{q_{n-1}(\omega)}\right| q_{n-1}^{2}(\omega) .
\end{aligned}
$$

Then $p_{n}(\omega) / q_{n}(\omega)=\left[0, a_{1}(\omega), \ldots, a_{n}(\omega)\right](n \geq 0), u_{1}(\omega)=x_{1}(\omega)$ and $u_{n}(\omega)=x_{n}(\omega)+\left(y_{n-1}(\omega)\right)^{-1}$ if $n \geq 2$ (see $\left.[1, \S 4]\right)$.

It is well known that endowing $(\Omega, \mathscr{B})$ with Gauss's measure

$$
P(B)=\frac{1}{\log 2} \int_{B} \frac{d \omega}{1+\omega} \quad(B \in \mathscr{B}),
$$

$\left\{a_{j}: j \geq 1\right\}$ is a stationary sequence of random variables which has good properties of weak dependence. Note that $P\left(a_{j}=k\right)=(\log 2)^{-1} \log \left(1+(k(k+2))^{-1}\right)$ $(k \geq 1)$.

We shall consider the $\sigma$-algebras

$$
\mathscr{M}_{j l}= \begin{cases}\sigma\left(a_{j-l}, \ldots, a_{j+l}\right) & \text { if } j-l \geq 1, \\ \sigma\left(a_{1}, \ldots, a_{j+l}\right) & \text { if } j-l<1 .\end{cases}
$$

The following lemma is Corollary 3.4 of [8]. Lemma 2 is due to Lévy ([1, (4.12)]).

Lemma 1. Let $\left\{\eta_{j}: j \geq 1\right\}$ be a sequence of measurable real functions on $(\Omega, \mathscr{B})$, and $\left\{b_{n}: n \geq 1\right\} \subset(0, \infty)$. Assume

(a) $\sup _{j} E_{P} \eta_{j}^{2}<\infty$ and $E_{P} \eta_{j}=0$ for every $j \geq 1$.

(b) $\lim _{n} n b_{n}^{-2}=0$.

(c) $\sum_{l=1}^{\infty} \sup _{j} E_{P}^{1 / 2}\left(\eta_{j}-E_{P}\left(\eta_{j} \mid \mathscr{M}_{j l}\right)\right)^{2}<\infty$

Then $\max _{1 \leq i \leq n}\left|b_{n}^{-1} \sum_{j=1}^{i} \eta_{j}\right| \rightarrow 0$ in measure.

Lemma 2. If $n \geq 2, y=\left[k_{n-1}, \ldots, k_{1}\right]$ with $k_{1}, \ldots, k_{n-1} \in \mathbf{N}^{*}$ and $1 \leq a<$ $b$, then

$$
\lambda\left(a<x_{n} \leq b \mid y_{n-1}=y\right)=\lambda((a, b]) \frac{y(y+1)}{(a y+1)(b y+1)} .
$$

After these preliminaries, we proceed to prove the theorem. From now on fix a function $f$ as in the statement. 
(I) Convergence of $\xi_{n}$

Step 1. Let $n \geq 1$ and $\omega \in \Omega$. It is well known that

$$
\begin{aligned}
& \left|q^{\prime} \omega-p^{\prime}\right|<\left(2 q^{\prime}\right)^{-1}, \text { g.c.d. }\left(p^{\prime}, q^{\prime}\right)=1, \text { implies } p^{\prime}=p_{k}(\omega), \\
& q^{\prime}=q_{k}(\omega) \text { for some } k \geq 0 .
\end{aligned}
$$

Then, if we define

$$
r_{j}(\omega)=\#\left\{r \in \mathbf{N}^{*}: r<\left(f\left(r q_{j-1}(\omega)\right) u_{j}(\omega)\right)^{1 / 2}\right\} \quad(j \geq 1),
$$

we have

$$
\begin{aligned}
\Lambda_{q_{n}(\omega)-1}(\omega) & =\#\left\{(k, r) \in \mathbf{N} \times \mathbf{N}^{*}:\left|r q_{k}(\omega) \omega-r p_{k}(\omega)\right|<\frac{f\left(r q_{k}(\omega)\right)}{r q_{k}(\omega)}\right. \\
& \left.1 \leq r q_{k}(\omega)<q_{n}(\omega)\right\} \\
& =\sum_{k=0}^{n-1} r_{k+1}(\omega) ;
\end{aligned}
$$

for the proof of the second equality, use the fact that

$$
\left|q_{k}(\omega) \omega-p_{k}(\omega)\right|=\left(x_{k+1}(\omega) q_{k}(\omega)+q_{k-1}(\omega)\right)^{-1} \quad \text { if } k \geq 1,
$$

and

$$
r<\left(\frac{1}{2} u_{k+1}(\omega)\right)^{1 / 2}, k \geq 0, \text { implies } r q_{k}(\omega)<q_{k+1}(\omega)
$$

(observe that if $k \geq 1$ and $r^{2}<(1 / 2) u_{k+1}$ then $\left(r q_{k}\right)^{2}<(1 / 2)\left(x_{k+1} q_{k}^{2}+q_{k} q_{k-1}\right)$ $\left.\leq a_{k+1} q_{k}^{2}+(1 / 2) q_{k} q_{k-1}<\left(a_{k+1} q_{k}+q_{k-1}\right)^{2}=q_{k+1}^{2}\right)$.

Define the r.v. $\tau_{n}: \Omega \rightarrow \mathbf{N}^{*}$ by

$$
\tau_{n}(\omega)=k \quad \text { if } q_{k-1}(\omega) \leq n<q_{k}(\omega) \quad(k=1,2, \ldots ; n \geq 1) .
$$

Therefore we have, for every $\omega \in \Omega$,

$$
\Lambda_{n}(\omega)=\sum_{j=1}^{\tau_{n}(\omega)} r_{j}(\omega)-\beta_{n}(\omega)
$$

where

$$
\begin{aligned}
\beta_{n}(\omega) & :=\#\left\{(p, q) \in \mathbf{N} \times \mathbf{N}^{*}:|q \omega-p|<\frac{f(q)}{q}, n<q<q_{\tau_{n}}(\omega)\right\} \\
& \leq\left(\frac{3}{2} a_{\tau_{n}}(\omega)\right)^{1 / 2}
\end{aligned}
$$

(we write $q_{\tau_{n}(\omega)}(\omega)=q_{\tau_{n}}(\omega)$, etc.). In order to prove the inequality in (6), note that if $(p, q)$ belongs to the set defining $\beta_{n}$ then, by (1) and (3), $p=r p_{\tau_{n}-1}(\omega)$, $q=r q_{\tau_{n}-1}(\omega)$ with a positive integer $r<\left((1 / 2) u_{\tau_{n}}(\omega)\right)^{1 / 2}$. 
Step 2. From now on fix $\rho \ll \lambda$. By [8, Example 2.14.1] we know that, writing

$$
\xi_{n}^{(1)}(t)=\left(\frac{\log 2}{c n \log n}\right)^{1 / 2} \sum_{j \leq[n t]}\left\{\left(c a_{j}\right)^{1 / 2}-E_{P}\left(c a_{1}\right)^{1 / 2}\right\} \quad(t \in[0,1]),
$$

$\mathscr{L}_{\rho}\left(\xi_{n}^{(1)}\right) \rightarrow{ }_{w} W$.

In place of $r_{j}$ we will deal first with $\tilde{r}_{j}$ which is defined by

$$
\tilde{r}_{j}(\omega)=\#\left\{r \in \mathbf{N}^{*}: r<\left(f\left(r \gamma_{j}\right) u_{j}(\omega)\right)^{1 / 2}\right\} \quad(j \geq 1),
$$

where $\gamma_{1}=1, \gamma_{j}=\left[\left(2^{1 / 2}\right)^{j-2}\right]$ if $j \geq 2$. We deduce from (7) that if

$$
\xi_{n}^{(2)}(t)=\left(\frac{\log 2}{c n \log n}\right)^{1 / 2} \sum_{j \leq[n t]}\left\{\tilde{r}_{j}-E_{P} \tilde{r}_{j}\right\}
$$

$\left(E_{P} \tilde{r}_{j} \leq E_{P}\left((3 / 2) a_{j}\right)^{1 / 2}<\infty\right)$, we have $\mathscr{L}_{\rho}\left(\xi_{n}^{(2)}\right) \rightarrow_{w} W$; for this purpose, it is sufficient to show that if

$$
g_{j}=\tilde{r}_{j}-\left(c a_{j}\right)^{1 / 2}, \quad \eta_{j}=g_{j}-E_{P} g_{j}, \quad j \geq 1
$$

(we shall see below that $E_{P}\left|g_{j}\right|<\infty$ ), then $\left\{\eta_{j}\right\}$ satisfies (a) and (c) of Lemma 1.

In order to verify (a) it is enough to prove that $\sup _{j \geq 1} E_{P} g_{j}^{2}<\infty$. First, we observe that for every $j \geq 1$ and $\omega \in \Omega$, if $\tilde{r}_{j}(\omega) \geq 1$ we have

$$
\tilde{r}_{j}(\omega)=\max \left\{r \in \mathbf{N}^{*}: r<\left(f\left(r \gamma_{j}\right) u_{j}(\omega)\right)^{1 / 2}\right\} ;
$$

in fact, since $f$ is nonincreasing, if $r, r^{\prime}$ are positive integers, $r^{\prime}<r$, and $r$ belongs to the set defining $\tilde{r}_{j}(\omega)$, then $r^{\prime}$ also belongs to that set. Thus we have, for each $j \geq 1$,

$$
\tilde{r}_{j}(\omega)=0 \Leftrightarrow u_{j}(\omega) \leq \frac{1}{f\left(\gamma_{j}\right)}
$$

and

$\left(9^{\prime \prime}\right) \quad$ for every $r \in \mathbf{N}^{*}, \quad \tilde{r}_{j}(\omega)=r \Leftrightarrow \frac{r^{2}}{f\left(r \gamma_{j}\right)}<u_{j}(\omega) \leq \frac{(r+1)^{2}}{f\left((r+1) \gamma_{j}\right)}$.

These relations show that $\left|g_{j}\right| \leq 1$ if $\tilde{r}_{j}=0$ and that

$$
-1 \leq g_{j} \leq\left(f\left(\left[\left(c a_{j}\right)^{1 / 2}\right]-1\right) u_{j}\right)^{1 / 2}-\left(c a_{j}\right)^{1 / 2} \text { if } \tilde{r}_{j} \geq 1 .
$$

Then

$$
\left|g_{j}\right| \leq 3\left(2 c^{1 / 2}\right)^{-1} a_{j}^{1 / 2}\left\{f\left(\left[\left(c a_{j}\right)^{1 / 2}\right]-1\right)-c\right\}+1
$$

and (a) of Lemma 1 holds if

$$
\sum_{k=1}^{\infty}\left\{f\left(\left[(c k)^{1 / 2}\right]-1\right)-c\right\}^{2} \frac{1}{k}<\infty
$$

$\left(P\left(a_{1}=k\right) \sim(\log 2)^{-1} k^{-2}\right)$; but this follows from the hypothesis on $f$. 
In what follows we will write $\tilde{r}_{j l}=E_{P}\left(\tilde{r}_{j} \mid \mathscr{M}_{j l}\right)$; note that $\eta_{j}-E_{P}\left(\eta_{j} \mid \mathscr{M}_{j l}\right)=$ $\tilde{r}_{j}-\tilde{r}_{j l}$. Define

$$
A_{j l}=\left\{u_{j} \leq \frac{1}{f\left(\gamma_{j}\right)}-\frac{6}{2^{l}}\right\} \cup \bigcup_{r=1}^{\infty}\left\{\frac{r^{2}}{f\left(r \gamma_{j}\right)}+\frac{6}{2^{l}}<u_{j} \leq \frac{(r+1)^{2}}{f\left((r+1) \gamma_{j}\right)}-\frac{6}{2^{l}}\right\} .
$$

It holds that $\tilde{r}_{j}=\tilde{r}_{j l}$ a.s. on $A_{j l}$. In order to see this, take positive integers $k_{j-l}, \ldots, k_{j+l}$ and $\omega \in A_{j l} \cap \Delta, \omega^{\prime} \in \Delta$, where

$$
\Delta=\Delta_{j l}\left(k_{j-l}, \ldots, k_{j+l}\right)= \begin{cases}\left\{a_{j-l}=k_{j-l}, \ldots, a_{j+l}=k_{j+l}\right\} & \text { if } j-l \geq 1, \\ \left\{a_{1}=k_{1}, \ldots, a_{j+l}=k_{j+l}\right\} & \text { if } j-l<1 ;\end{cases}
$$

we have $\tilde{r}_{j}(\omega)=\tilde{r}_{j}\left(\omega^{\prime}\right)$ by the definition of $A_{j l},(9)$, and the fact that $\mid u_{j}(\omega)-$ $u_{j}\left(\omega^{\prime}\right) \mid<62^{-l}$ (see, for example, [8, (4.4)]).

Therefore, since $\tilde{r}_{j} \leq\left((3 / 2) a_{j}\right)^{1 / 2}$,

$$
E_{P}\left(\eta_{j}-E_{P}\left(\eta_{j} \mid \mathscr{M}_{j l}\right)\right)^{2}=E_{P}\left(\tilde{r}_{j}-\tilde{r}_{j l}\right)^{2} \leq 6 \int_{A_{j l}^{c}} a_{j} d P .
$$

Now observe that

$$
A_{j l}^{c}=\bigcup_{r=1}^{\infty} J_{j l r}
$$

where

$$
J_{j l r}=\left\{\frac{r^{2}}{f\left(r \gamma_{j}\right)}-\frac{6}{2^{l}}<u_{j} \leq \frac{r^{2}}{f\left(r \gamma_{j}\right)}+\frac{6}{2^{l}}\right\} \quad\left(r \in \mathbf{N}^{*}\right) .
$$

Assume $l \geq 2$. We will show that for some constant $K$,

$$
\lambda\left(J_{j l r}\right) \leq K 2^{-l} r^{-4} \text {. }
$$

Suppose $j \geq 2$ and take $r \in \mathbf{N}^{*}$. Writing $\alpha(r)=r^{2}\left(f\left(r \gamma_{j}\right)\right)^{-1}$, we have

$$
\begin{aligned}
\lambda\left(J_{j l r}\right)= & \sum \lambda\left(1<x_{j} \leq \alpha(r)+62^{-l}-y^{-1} \mid y_{j-1}=y\right) \lambda\left(y_{j-1}=y\right) \\
& +\sum \lambda\left(\alpha(r)-62^{-l}-y^{-1}<x_{j} \leq \alpha(r)+62^{-l}-y^{-1} \mid y_{j-1}=y\right) \\
& \times \lambda\left(y_{j-1}=y\right) \\
= & A+B \text { (say), }
\end{aligned}
$$

where the first sum is taken over all possible values $y$ of $y_{j-1}$ which satisfy $\alpha(r)-62^{-l}-y^{-1}<1$ (this implies that $\alpha(r) \geq r^{4}$ ), and in the second one $\alpha(r)-62^{-l}-y^{-1} \geq 1$ (in this case $\alpha(r)-62^{-l} \geq(1 / 2) r^{2}$ because $\left.l \geq 2\right)$. By Lemma 2,

$$
\begin{aligned}
A & =\sum\left(\alpha(r)-y^{-1}-1+62^{-l}\right)\left(\alpha(r)+62^{-l}\right)^{-1} \lambda\left(y_{j-1}=y\right) \\
& \leq 122^{-l} r^{-4} \\
B & =122^{-l} \sum\left(1+y^{-1}\right)\left(\left(\alpha(r)-62^{-l}\right)\left(\alpha(r)+62^{-l}\right)\right)^{-1} \lambda\left(y_{j-1}=y\right) \\
& \leq 242^{-l} r^{-4} .
\end{aligned}
$$


Hence $\lambda\left(J_{j l r}\right) \leq 362^{-l} r^{-4}$ when $j \geq 2$ and we obtain a similar bound for $j=1$ using the fact that $\mathscr{L}_{P}\left(x_{1}\right)(d t)=I_{(1, \infty)}(t)(t(t+1) \log 2)^{-1} d t$. This proves (12).

On the other hand, since $a_{j} \leq u_{j}$, there exists a constant $K^{\prime}$ such that

$$
a_{j} \leq K^{\prime} r^{2} \text { on } J_{j l r} \text {. }
$$

From (10)-(13), which are valid for $j \geq 1$ and $l \geq 2$, we conclude that $\left\{\eta_{j}\right\}$ satisfies (c) of Lemma 1 (for $j \geq 1, l=1, E_{P}\left(\eta_{j}-E_{P}\left(\eta_{j} \mid \mathscr{M}_{j 1}\right)\right)^{2} \leq$ $\left.4 \sup _{j} E_{P} \eta_{j}^{2}<\infty\right)$. Therefore $\mathscr{L}_{\rho}\left(\xi_{n}^{(2)}\right) \rightarrow{ }_{w} W$.

Step 3. We want to replace $E_{P} \tilde{r}_{j}$ in $\xi_{n}^{(2)}$ by a constant. First we prove that

$$
\left|E_{P} \tilde{r}_{j}-E_{\lambda} \tilde{r}_{j}\right|=O\left(s_{1}^{j}\right) \text { for some } s_{1} \in(0,1) .
$$

The preceding step shows that for some constant $K, E_{P}^{1 / 2}\left(\tilde{r}_{j}-\tilde{r}_{j l}\right)^{2} \leq$ $K\left(2^{1 / 2}\right)^{-l}$. Moreover, arguing as in the proof of [8, (4.15)], we have $\mid E_{P} \tilde{r}_{j l}-$ $E_{\lambda} \tilde{r}_{j l} \mid \leq K^{\prime}\left(s^{\prime}\right)^{j-l}$ if $j>l$, for some constants $K^{\prime}$ and $s^{\prime} \in(0,1)$. Applying these two inequalities (with $l_{j}=[j / 2]$ ) we deduce (14).

Using (9) and Lemma 2 we have, for $j \geq 2$ and any positive integer $r$ (with the notation $\alpha(r)$ of the preceding step),

$$
\begin{aligned}
\lambda\left(\tilde{r}_{j} \geq r\right) & =\sum_{y} \lambda\left(x_{j}>\alpha(r)-y^{-1} \mid y_{j-1}=y\right) \lambda\left(y_{j-1}=y\right) \\
& =r^{-2} f\left(r \gamma_{j}\right) E_{\lambda}\left(1+y_{j-1}^{-1}\right)
\end{aligned}
$$

then

$$
E_{\lambda} \tilde{r}_{j}=\left\{\sum_{r=1}^{\infty} r^{-2} f\left(r \gamma_{j}\right)\right\} E_{\lambda}\left(1+y_{j-1}^{-1}\right) \quad(j \geq 2) .
$$

Denoting by $m_{j}$ this quantity for $f \equiv c$, we can assert that

$$
m_{j}=c \frac{\pi^{2}}{6} E_{\lambda}\left(1+y_{j-1}^{-1}\right)=c \frac{\pi^{2}}{6}\left(\frac{1}{\log 2}+O\left(s_{2}^{j}\right)\right) \quad \text { for some } s_{2} \in(0,1) .
$$

The last relation follows from a theorem of Lévy which is used in the proof of [8, Lemma 4.5(b)] (or apply directly that result).

Now if we show that

$$
(n \log n)^{-1 / 2} \sum_{j=1}^{n}\left(f\left(\gamma_{j}\right)-c\right) \rightarrow 0 \quad \text { as } n \rightarrow \infty
$$

then (14)-(16) will yield, by noting that $\left|E_{\lambda} \tilde{r}_{j}-m_{j}\right| \leq\left(\pi^{2} / 3\right)\left(f\left(\gamma_{j}\right)-c\right)$ for $j \geq 2$ ( $f$ is nonincreasing),

$$
(n \log n)^{-1 / 2} \sum_{j=1}^{n}\left|E_{P} \tilde{r}_{j}-(6 \log 2)^{-1} c \pi^{2}\right| \rightarrow 0
$$


as $n \rightarrow \infty$. This will imply that, denoting

$$
\xi_{n}^{(3)}(t)=\left(\frac{\log 2}{c n \log n}\right)^{1 / 2} \sum_{j \leq[n t]}\left\{\tilde{r}_{j}-\frac{c \pi^{2}}{6 \log 2}\right\},
$$

$\mathscr{L}_{\rho}\left(\xi_{n}^{(3)}\right) \rightarrow_{w} W$

In order to prove (16), first observe that, given a sequence of positive integers $\{j(n)\}$ (to be defined later), the quantity there is

$$
O\left(j(n)(n \log n)^{-1 / 2}\right)+O\left((n / \log n)^{1 / 2}\left(f\left(\gamma_{j(n)}\right)-c\right)\right)
$$

Write $f(q)=c+\varepsilon(q) \log (\log q)(\log q)^{-1}\left(q \in \mathbf{N}^{*}\right)$ where $\lim _{q} \varepsilon(q)=0$. There exist $\delta(n) \rightarrow 0$ such that, if we define $j(n)=\left[\delta(n)(n \log n)^{1 / 2}\right]$ then $j(n) \rightarrow \infty$ and $\delta(n)^{-1} \varepsilon\left(\gamma_{j(n)}\right) \rightarrow 0$ as $n \rightarrow \infty$ (first choose $n_{1}<n_{2}<\cdots$ such that for all $n \geq n_{i}$ it holds that $i \varepsilon\left(\gamma_{\left[i^{-1}(n \log n)^{1 / 2}\right]}\right) \leq i^{-1}$ and $i^{-1}(n \log n)^{1 / 2} \geq i$; then define $\delta(n)=i^{-1}$ if $\left.n_{i} \leq n<n_{i+1}\right)$. This implies (16) since the second term in (17) is $O\left(\delta(n)^{-1} \varepsilon\left(\gamma_{j(n)}\right)\right)$ for this $j(n)$.

As indicated above, we deduce the convergence of $\mathscr{L}_{\rho}\left(\xi_{n}^{(3)}\right)$.

Step 4. The $r_{j}$ satisfy similar relations to (9):

$$
r_{j}(\omega)=0 \Leftrightarrow u_{j}(\omega) \leq \frac{1}{f\left(q_{j-1}(\omega)\right)}
$$

and, for every $r \in \mathbf{N}^{*}$,

$$
r_{j}(\omega)=r \Leftrightarrow \frac{r^{2}}{f\left(r q_{j-1}(\omega)\right)}<u_{j}(\omega) \leq \frac{(r+1)^{2}}{f\left((r+1) q_{j-1}(\omega)\right)} .
$$

Suppose $j \geq 2$. From elementary properties of continued fractions, $q_{j-1}=$ $\tilde{q}_{j-1}\left(y_{j-1}\right)$ where $\tilde{q}_{j-1}$ is a function defined on the set of possible values of $y_{j-1}$. Given $r \in \mathbf{N}^{*}$, write $\alpha(r, y)=r^{2}\left(f\left(r \tilde{q}_{j-1}(y)\right)\right)^{-1}$ if $y$ is a value of $y_{j-1}$; the preceding relations and Lemma 2 show that

$$
\begin{aligned}
\lambda\left(r_{j} \geq r\right) & =\sum_{y} \lambda\left(x_{j}>\alpha(r, y)-y^{-1} \mid y_{j-1}=y\right) \lambda\left(y_{j-1}=y\right) \\
& =r^{-2} E_{\lambda}\left(f\left(r q_{j-1}\right)\left(1+y_{j-1}^{-1}\right)\right) .
\end{aligned}
$$

Therefore, since $r_{j} \leq \tilde{r}_{j}$, which is due to the monotonicity of $f$ and the fact that $q_{j-1} \geq \gamma_{j}($ see $[1,(4.8)])$, we have

$$
\begin{aligned}
E_{\lambda}\left|\tilde{r}_{j}-r_{j}\right| & =\sum_{r=1}^{\infty} r^{-2} E_{\lambda}\left(\left\{f\left(r \gamma_{j}\right)-f\left(r q_{j-1}\right)\right\}\left(1+y_{j-1}^{-1}\right)\right) \\
& \leq\left(\pi^{2} / 3\right)\left(f\left(\gamma_{j}\right)-c\right) .
\end{aligned}
$$


Hence, by using (16) we conclude that $(n \log n)^{-1 / 2} \sum_{j=1}^{n}\left|\tilde{r}_{j}-r_{j}\right| \rightarrow 0$ in measure, which in turn yields that $\mathscr{L}_{\rho}\left(\xi_{n}^{(4)}\right) \rightarrow_{w} W$, where

$$
\xi_{n}^{(4)}(t)=\left(\frac{\log 2}{c n \log n}\right)^{1 / 2} \sum_{j \leq[n t]}\left\{r_{j}-\frac{c \pi^{2}}{6 \log 2}\right\} .
$$

Step 5. By a well-known theorem of Khintchine, $\lim _{k} k^{-1} \log q_{k}=B:=\pi^{2} /$ $(12 \log 2)$ a.e. This and (4) give

$$
\lim _{n} \frac{\tau_{n}}{\log n}=B^{-1} \text { a.e. }
$$

Now we claim

$$
\eta_{n}:=(\log n \log (\log n))^{-1 / 2} \max _{1 \leq k \leq n} a_{\tau_{k}}^{1 / 2} \rightarrow 0 \quad \text { in measure. }
$$

For every $\varepsilon>0$, since $\tau_{k} \leq \tau_{n}$ if $k \leq n$,

$$
\begin{aligned}
P\left(\eta_{n}>\varepsilon\right) \leq & P\left(\tau_{n}>(1+\varepsilon) B^{-1} \log n\right) \\
& +P\left((\log n \log (\log n))^{-1 / 2} \max _{j \leq(1+\varepsilon) B^{-1} \log n} a_{j}^{1 / 2}>\varepsilon\right)
\end{aligned}
$$

and the second term on the right is bounded by

$$
(1+\varepsilon) B^{-1}(\log n) P\left(a_{1}>\varepsilon^{2} \log n \log (\log n)\right) ;
$$

then (18) implies (19) because $P\left(a_{1}>x\right) \sim(x \log 2)^{-1}$ as $x \rightarrow \infty$.

We also need

$$
\zeta_{n}:=(\log n \log (\log n))^{-1 / 2} \max _{1 \leq k \leq n}\left|B \tau_{k}-\log k\right| \rightarrow 0 \text { in measure. }
$$

First observe that, since $q_{\tau_{k}-1} \leq k<q_{\tau_{k}}, 0 \leq \log q_{\tau_{k}}-\log k \leq \log y_{\tau_{k}} \leq$ $\log \left(a_{\tau_{k}}+1\right) \leq a_{\tau_{k}}^{1 / 2}$, and we get $\left|B \tau_{k}-\log k\right| \leq\left|B \tau_{k}-\log q_{\tau_{k}}\right|+a_{\tau_{k}}^{1 / 2}$. Moreover, if $Y_{n}(t)=\tau_{n}^{-1 / 2}\left(\log q_{\left[t \tau_{n}\right]}-\left[t \tau_{n}\right] B\right) \quad(t \in[0,1])$, we have

$$
\max _{k \leq n}\left|\log q_{\tau_{k}}-B \tau_{k}\right|=\tau_{n}^{1 / 2} \sup _{t \in[0,1]}\left|Y_{n}(t)\right|
$$

and (20) follows from (18), (19), and a theorem of Billingsley [2, p. 194 and Theorem 17.1].

We claim that if

$$
\xi_{n}^{(5)}(t)=\left(\frac{\pi^{2} / 12}{c \log n \log (\log n)}\right)^{1 / 2} \sum_{j=1}^{\tau_{\left.n^{t}\right]}}\left\{r_{j}-\frac{c \pi^{2}}{6 \log 2}\right\},
$$

then $\mathscr{L}_{\rho}\left(\xi_{n}^{(5)}\right) \rightarrow_{w} W$. Here we will use the notation and results of [2, §17]. Define

$$
\Phi_{n}(t, \omega)= \begin{cases}{[\log n]^{-1} \tau_{\left[n^{t}\right]}(\omega),} & \text { if }[\log n]^{-1} \tau_{n}(\omega) \leq 1, \\ t B^{-1}, & \text { otherwise }\end{cases}
$$


and $g(t)=t B^{-1}(t \in[0,1], \omega \in \Omega)$. We have $\Phi_{n}(\cdot, \omega) \in D_{0}$ for each $\omega, g \in D_{0}$, and $\Phi_{n}$ converges in $\rho$-measure to $g$ in $D_{0}$; this follows from (20) since

$$
\sup _{t \in[0,1]}\left|\Phi_{n}(t, \cdot)-g(t)\right| \leq[\log n]^{-1}\left\{\max _{1 \leq k \leq n}\left|\tau_{k}-B^{-1} \log k\right|+B^{-1}\right\} .
$$

Starting from the weak convergence of $\mathscr{L}_{\rho}\left(\xi_{[\log n]}^{(4)}, \Phi_{n}\right)$ (use [2, Theorem 4.4]), noting that if $\omega$ satisfies $[\log n]^{-1} \tau_{n}(\omega) \leq 1$ and $t \in[0,1]$,

$$
\left(\xi_{[\log n]}^{(4)} \circ \Phi_{n}\right)(t, \omega)=\left(\frac{\log 2}{c[\log n] \log ([\log n])}\right)^{1 / 2} \sum_{j=1}^{\tau_{\left[n^{t}\right]}(\omega)}\left\{r_{j}(\omega)-\frac{c \pi^{2}}{6 \log 2}\right\},
$$

and using (18), we can argue as in [2, proof of Theorem 17.1] to conclude that $\mathscr{L}_{\rho}\left(\xi_{n}^{(5)}\right) \rightarrow_{w} W$.

In order to complete the proof, observe that by (5) and (6)

$$
\sup _{t \in[0,1]}\left|\xi_{n}(t)-\xi_{n}^{(5)}(t)\right| \leq\left(\pi^{2} /(12 c)\right)^{1 / 2}\left((3 / 2)^{1 / 2} \eta_{n}+2 c \zeta_{n}\right) \quad \text { a.e. }
$$

and use (19) and (20).

(II) Convergence of $\xi_{n}^{+}$. Minor modifications in (I) are needed. We merely remark that since a convergent $p_{k}(\omega) / q_{k}(\omega)$ is less than $\omega$ if and only if $k$ is even, (5) is true for $\Lambda_{n}^{+}$if in the sum of the right member $j$ runs through odd values and $\beta_{n}$ is suitably defined; then the inequality in (6) also holds.

\section{ACKNOWLEDGMENT}

Part of this work was done while visiting the Département de Mathématique at Université Louis Pasteur, Strasbourg. I am grateful to that institution for its hospitality.

\section{REFERENCES}

1. P. Billingsley, Ergodic theory and information, Wiley, New York, 1965.

2. _ Convergence of probability measures, Wiley, New York, 1968.

3. P. Erdös, Some results on diophantine approximation, Acta Arith. 5 (1959), 359-369.

4. S. Lang, Introduction to diophantine approximations, Addison-Wesley, Reading, MA, 1966.

5a. W. J. LeVeque, On the frequency of small fractional parts in certain real sequences, Trans. Amer. Math. Soc. 87 (1958), 237-260.

5b. _ On the frequency of small fractional parts in certain real sequences. II, Trans. Amer. Math. Soc. 94 (1960), 130-149.

6. W. Philipp, Mixing sequences of random variables and probabilistic number theory, Mem. Amer. Math. Soc. 114 (1971).

7. W. Philipp and W. Stout, Almost sure invariance principles for partial sums of weakly dependent random variables, Mem. Amer. Math. Soc. 161 (1975). 
8. J. D. Samur, On some limit theorems for continued fractions, Trans. Amer. Math. Soc. $\mathbf{3 1 6}$ (1989), 53-79.

9. W. M. Schmidt, Diophantine approximation, Lecture Notes in Math., vol. 785, Springer-Verlag, Berlin, 1980.

Departamento de Matemática, Facultad de Ciencias Exactas, Universidad Nacional de la Plata, Casilla de Correo 172, 1900 la Plata, Argentina 